\title{
Article \\ Predicting Prognosis of Breast Cancer Patients with Brain Metastases in the BMBC Registry-Comparison of Three Different GPA Prognostic Scores
}

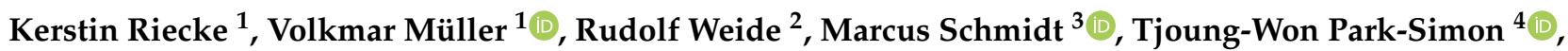 \\ Volker Möbus ${ }^{5}$, Christoph Mundhenke ${ }^{6,7}$ (D), Arkadius Polasik ${ }^{8}$, Kristina Lübbe ${ }^{9}$ (D), Tobias Hesse ${ }^{10}$, \\ Elena Laakmann ${ }^{1}$, Marc Thill ${ }^{11}$, Peter A. Fasching ${ }^{12}$, , Carsten Denkert ${ }^{13}$, Tanja Fehm ${ }^{14}$, \\ Valentina Nekljudova ${ }^{15}$, Julia Rey ${ }^{15}$, Sibylle Loibl ${ }^{15}$ and Isabell Witzel ${ }^{1, *(D)}$
}

check for

updates

Citation: Riecke, K.; Müller, V.; Weide, R.; Schmidt, M.; Park-Simon, T.-W.; Möbus, V.; Mundhenke, C.; Polasik, A.; Lübbe, K.; Hesse, T.; et al. Predicting Prognosis of Breast Cancer Patients with Brain Metastases in the BMBC Registry-Comparison of Three Different GPA Prognostic Scores. Cancers 2021, 13, 844 https://doi.org/10.3390/ cancers13040844

Academic Editor: Simon S. Lo

Received: 14 January 2021

Accepted: 12 February 2021

Published: 17 February 2021

Publisher's Note: MDPI stays neutral with regard to jurisdictional claims in published maps and institutional affiliations.

Copyright: (c) 2021 by the authors. Licensee MDPI, Basel, Switzerland. This article is an open access article distributed under the terms and conditions of the Creative Commons Attribution (CC BY) license (https:// creativecommons.org/licenses/by/ $4.0 /)$.
1 Department of Gynecology, University Medical Center Hamburg-Eppendorf, Martinistraße 52, 20246 Hamburg, Germany; k.riecke@uke.de (K.R.); v.mueller@uke.de (V.M.); e.laakmann@uke.de (E.L.)

2 Oncological Outpatient Department, Neversstraße 5, 56068 Koblenz, Germany; weide@onkologie-koblenz.de

3 Department of Gynecology, University Medical Center of the Johannes Gutenberg University Mainz, Langenbeckstr. 1, 55131 Mainz, Germany; Marcus.Schmidt@unimedizin-mainz.de

4 Department of Gynecology, Hannover Medical School, Carl-Neuberg-Straße 1, 30625 Hannover, Germany; Park-Simon.Tjoung-Won@mh-hannover.de

5 Department of Hematology and Oncology, University Hospital Frankfurt am Main, Theodor-Stern-Kai 7, 60590 Frankfurt am Main, Germany; Volker.Moebus@kgu.de

6 Clinic Bayreuth, Preuschwitzer Str. 101, 95445 Bayreuth, Germany; christoph.mundhenke@klinikum-bayreuth.de

7 Department of Gynecology and Obstetrics, University Medical Center Schleswig-Holstein, Arnold-Heller-Straße 3, 24105 Kiel, Germany

8 Department of Gynecology and Obstetrics, University Medical Center Ulm, Prittwitzstr. 43, 89075 Ulm, Germany; arkadius.polasik@uniklinik-ulm.de

9 Breast Center, Diakovere Henriettenstift, Schwemannstraße 17, 30559 Hannover, Germany; Kristina.Luebbe@diakovere.de

10 Department of Gynecology, Agaplesion Diakonie Clinic Rotenburg, Elise-Averdieck-Straße 17, 27356 Rotenburg, Germany; Hesse@diako-online.de

11 Department of Gynecology and gynecologic Oncology, Agaplesion Markus Hospital, Wilhelm-Epstein-Str. 4, 60341 Frankfurt am Main Frankfurt, Germany; Marc.Thill@fdk.info

12 Department of Gynecology and Obstetrics, University Hospital Erlangen, Comprehensive Cancer Center Erlangen-EMN, Friedrich-Alexander University Erlangen-Nuremberg, Universitätsstraße 21-23, 91054 Erlangen, Germany; Peter.Fasching@uk-erlangen.de

13 Institute of Pathology, University Hospital Marburg, Baldingerstraße, 35043 Marburg, Germany; carsten.denkert@uni-marburg.de

14 Department of Gynecology and Obstetrics, University Hospital Düsseldorf, Moorenstr. 5, 40225 Düsseldorf, Germany; Tanja.Fehm@med.uni-duesseldorf.de

15 German Breast Group, Martin-Behaim-Straße 12, 63263 Neu-Isenburg, Germany; Valentina.Nekljudova@gbg.de (V.N.); Julia.Rey@gbg.de (J.R.); Sibylle.Loibl@gbg.de (S.L.)

* Correspondence: i.witzel@uke.de; Tel.: +49-40-7410-52510

Simple Summary: The incidence of brain metastases from breast cancer is increasing and the treatment is still a major challenge. Several scores have been developed in order to estimate the prognosis of patients with brain metastases by objective criteria. Here, we validated all three published graded-prognostic-assessment (GPA)-scores in a subcohort of 882 breast cancer patients with brain metastases in the Brain Metastases in the German Breast Cancer (BMBC) registry. Although all three available GPA-scores were associated with OS, they all show limitations mainly in predicting short-term (below 3 months) survival but also in long-term (above 12 months) survival. We discuss the test performances of all scores in our work and provide evidence how physicians should use them as a tool to select patients for different treatment options.

Abstract: Several scores have been developed in order to estimate the prognosis of patients with brain metastases (BM) by objective criteria. The aim of this analysis was to validate all three published graded-prognostic-assessment (GPA)-scores in a subcohort of 882 breast cancer (BC) patients with BM 
in the Brain Metastases in the German Breast Cancer (BMBC) registry. The median age at diagnosis of BM was 57 years. All in all, $22.3 \%$ of patients $(n=197)$ had triple-negative, $33.4 \%(n=295)$ luminal A like, $25.1 \%(n=221)$ luminal B/HER2-enriched like and 19.2\% $(n=169)$ HER2 positive like BC. Age $\geq 60$ years, evidence of extracranial metastases (ECM), higher number of BM, triple-negative subtype and low Karnofsky-Performance-Status (KPS) were all associated with worse overall survival (OS) in univariate analysis ( $p<0.001$ each). All three GPA-scores were associated with OS. The breast-GPA showed the highest probability of classifying patients with survival above 12 months in the best prognostic group (specificity $68.7 \%$ compared with $48.1 \%$ for the updated breast-GPA and $21.8 \%$ for the original GPA). Sensitivities for predicting 3 months survival were very low for all scores. In this analysis, all GPA-scores showed only moderate diagnostic accuracy in predicting the OS of BC patients with BM.

Keywords: brain metastases; breast cancer; prognostic scores

\section{Introduction}

The incidence of brain metastases (BM) from breast cancer $(\mathrm{BC})$ is increasing and the management and treatment of those patients continue to be a major challenge [1]. BM mostly occur as a late event of metastatic disease after several systemic treatments and their prognosis remains limited [2]. Despite the short median survival times in several cohorts of metastatic BC patients with BM ranging between 7 and 8 months, almost a quarter of patients live longer than 2 years [3]. Although a growing number of therapeutic options for systemic therapy emerge, the management and treatment of BM as well as selecting patients for different therapies remains unsatisfactory. In the past, several prognostic indices have been published in order to help identify patients with good prognosis, eligible for intensive treatment and those with limited prognosis who should be protected from overtreatment with systemic therapy causing side effects. Those patients might be treated better with supportive care in order to help improve their quality of life [4,5]. In 2008, Sperduto et al. established the graded prognostic assessment (GPA)-Score deriving from patients suffering from BM irrespective of their primary tumour localisation [6]. Due to the fact that prognosis differs between different subtypes of primary tumours [7], Sperduto et al. established the disease-specific Breast-GPA score in 2012 deriving from 400 BC patients including prognostic factors such as age, the Karnofsky performance status (KPS) and tumour subtype (Table 1) [8]. An updated version of this score adding the existence of extracranial metastase (ECM) and number of BM to the parameters of the Breast-GPA was published in 2020 in order to increase the test value of the existing Breast-GPA [9]. It has already been described in small cohorts that the Breast-GPA best identifies patients with bad prognosis defined as survival time below 3 months $[4,10]$.

This analysis aimed to examine the performance of the original GPA, the Breast-GPA and the updated Breast-GPA in a large cohort of BC patients with BM in the BMBC registry and to test the accuracy of all scores to predict short survival (defined as below 3 months) or long survival (defined as more than 12 months).

Table 1. Calculation of original graded-prognostic-assessment (GPA), Breast-GPA and updated Breast-GPA.

\begin{tabular}{cccc}
\hline GPA & 0 points & $\mathbf{0 . 5}$ points & $\mathbf{1 . 0}$ points \\
\hline Karnofsky & $\leq 60$ & $70-80$ & $90-100$ \\
\hline Number of BM & $\geq 4$ & $2-3$ & 1 \\
\hline ECM & yes & no \\
\hline Age & $\geq 60$ & $50-59$ & $<50$ \\
\hline & group 1: 0-1P; 2:1.5-2.5P; 3: 3.0P; 4: 3.5-4.0P \\
\hline
\end{tabular}


Table 1. Cont.

\begin{tabular}{ccccc}
\hline GPA & 0 points & 0.5 points & 1.0 points & \\
\hline Breast-GPA & 0 points & 0.5 points & 1.0 points & 1.5 points \\
\hline Karnofsky & $\leq 50$ & 60 & $70-80$ & $90-100$ \\
\hline Subtype & TNBC & n/a & LumA & HER2 \\
\hline Age & $\geq 60$ & $<60$ & n/a & n/a \\
\hline group 1:0-1 P.; 2:1.5-2.0 P.;3: 2.5-3.0 P.; 4:3.5-4.0 P. & \\
\hline Updated Breast-GPA & 0 points & 0.5 points & 1.0 points & 1.5 points \\
\hline Karnofsky & $\leq 60$ & $70-80$ & $90-100$ & $\mathrm{n} / \mathrm{a}$ \\
\hline Subtype & TNBC & n/a & LumA & HER2, LumB \\
\hline Age & $\geq 60$ & $<60$ & $\mathrm{n} / \mathrm{a}$ & $\mathrm{n} / \mathrm{a}$ \\
\hline Number of BM & $>1$ & $=1$ & $\mathrm{n} / \mathrm{a}$ & $\mathrm{n} / \mathrm{a}$ \\
\hline ECM & yes & no & \\
\hline
\end{tabular}

\section{Results}

\subsection{Patients' Characteristics}

A total of 882 patients were included in this analysis (Table 2). Patients' characteristics did not differ between the entire cohort of the BMBC registry and this subset of patients (data not shown).

Median age at the diagnosis of BM was 57 years. Tumour subtypes of the primary tumour were distributed as following: TNBC 22.3\% $(n=197)$, Luminal A $33.4 \%(n=295)$, Luminal B/HER2 enriched $25.1 \%(n=221)$, HER2 positive $19.2 \%(n=169)$. All in all, $26.5 \%$ of patients had one $(n=234), 27.8 \%$ had two or three $(n=245)$ and $45.7 \%$ had four and more $\mathrm{BM}(n=403)$. A further $7.3 \%$ of patients $(n=64)$ had leptomeningeal disease. Additionally, $15.6 \%$ of patients $(n=138)$ had no ECM. BM were mainly detected by magnetic resonance imaging (MRI) $(n=589,67.1 \%)$. Regarding local treatment, the majority of patients received local treatment of BM $(86.5 \%, n=763)$ either as radiotherapy $(n=544)$, surgery followed by radiotherapy $(n=185)$ or only surgery $(n=34)$. Systemic treatment options were chemotherapy ( $n=466,44.9 \%)$, endocrine therapy $(n=159,15.3 \%)$ or other therapies such as anti-HER2 treatments, bisphosphonates and bevacizumab ( $n=414,39.8 \%$ ) (Table S4, Table S5, Table S6). Most of the patients had a good performance status at diagnosis of BM (KPS 100\%: 13.5\%, 80-90\% 44.6\%, 60-70\%: 30.3\%, 40-50\%: 9.1\%, 10-30\%: 2.6\%).

Table 2. Patients' characteristics $(n=882)$.

\begin{tabular}{ccc}
\hline Parameter & Category & Number (\%) \\
\hline Age at first diagnosis of BC, years & Median & 53.0 \\
Age at diagnosis of BM, years & missing & $20.0,87.0$ \\
& Median & 0 \\
range & missing & 57.0 \\
Histological tumour type & ductal or & $25.0,90.0$ \\
& ductal-lobular-invasive & 0 \\
Biological subtype & lobular-invasive & $691(78.8)$ \\
(according to Sperduto 2012) & other & $60(6.8)$ \\
& missing & $126(14.4)$ \\
& Luminal A (HR+, HER2-) & 5 \\
& Luminal B /HER2 enriched & $197(22.3)$ \\
& (HR+, HER2+) & $295(33.4)$ \\
& & $221(25.1)$ \\
\hline
\end{tabular}


Table 2. Cont.

\begin{tabular}{|c|c|c|}
\hline Parameter & Category & Number (\%) \\
\hline \multirow{8}{*}{ KPS at diagnosis of BM } & HER2 (HR - , HER2+) & $169(19.2)$ \\
\hline & missing & 0 \\
\hline & $100 \%$ & $119(13.5)$ \\
\hline & $80-90 \%$ & $393(44.6)$ \\
\hline & $60-70 \%$ & $267(30.3)$ \\
\hline & $40-50 \%$ & $80(9.1)$ \\
\hline & $10-30 \%$ & $23(2.6)$ \\
\hline & missing & 0 \\
\hline \multirow[t]{4}{*}{ Number of BM } & 1 & $234(26.5)$ \\
\hline & $2-3$ & $245(27.8)$ \\
\hline & $\geq 4$ & $403(45.7)$ \\
\hline & missing & 0 \\
\hline \multirow[t]{5}{*}{ Diagnostic method to detect BM } & only clinical & $9(1.0)$ \\
\hline & CT w/o clinical & $209(23.8)$ \\
\hline & MRI w/o clinical & $589(67.1)$ \\
\hline & CT and MRI w/o clinical & $71(8.1)$ \\
\hline & missing & 4 \\
\hline \multirow[t]{4}{*}{ Local treatment of BM } & Surgery only & $34(4.5)$ \\
\hline & Radiotherapy only & $544(71.3)$ \\
\hline & Surgery and radiotherapy & $185(24.2)$ \\
\hline & missing & 119 \\
\hline \multirow[t]{3}{*}{ ECM } & no & $138(15.6)$ \\
\hline & yes & $744(84.4)$ \\
\hline & no & $814(92.7)$ \\
\hline \multirow[t]{2}{*}{ Leptomeningeal metastases } & yes & $64(7.3)$ \\
\hline & missing & 4 \\
\hline
\end{tabular}

\subsection{Survival Analysis}

The median OS in our cohort was 8.7 months. Median OS rates at 6-month were $57.4 \%$, at 1-, 2- and 3-year they were $41.3 \%, 24.3 \%$ and $16.6 \%$, respectively.

In univariate analysis, clinical variables like age (categorised in $<60 / \geq 60$ years), appearance of ECM, KPS, number of BM and biological subtype were all associated with survival (Table 3). Patients 60 years and older had a worse prognosis (median survival of 5 months (95\% CI 3.9-5.9) vs. 11.3 months (95\% CI 9.6-13.7), Hazard Ratio (HR) $1.53(1.3-1.7795 \% \mathrm{CI}, p<0.001)$. Evidence of ECM at the diagnosis of BM was associated with shorter OS (7.8 months (95\% CI 6.4-9.0; HR 1.67, 1.34-2.08 95\% CI, $p<0.001)$ vs. 15.6 months (95\% CI 9.2-13.6). The number of $\mathrm{BM}$ was prognostic with a median OS time of 14.1 months for patients with $1 \mathrm{BM}$ (95\% CI 10.3-19.2) followed by 9.7 months for 2-3 BM (95\% CI 6.1-12.3) and 6.2 months (95\% CI 5.1-7.2) for 4 or more BM (HR for $\geq 4$ BM 1.80 (95\% CI 1.5-2.16, $p<0.001)$. The tumour subtype of the primary tumour also had an influence on survival times. Patients with TNBC had the shortest median OS with 4.8 months (95\% CI 3.8-6.1), followed by Luminal A subtype with 6.0 months (95\% CI 5.0-7.9) and HER2 positive (ER-negative) subtype with 12.3 months (95\% CI 9.2-17.3), whereas patients with Luminal-B/HER2-enriched subtype had the longest median survival time of 16.0 months (95\% CI 13.0-21.7). Consecutively, luminal B/HER2-enriched patients had a significantly lower risk of death compared to patients with other subtypes ( $\mathrm{HR}=0.43,95 \% \mathrm{CI} 0.34,0.53, p<0.001)$. We also performed an analysis for the progression-free interval after diagnosis of $\mathrm{BM}$ and the time from diagnosis of ECM to BM according to breast cancer subtype and found that TNBC patients had the shortest progression-free interval since the first diagnosis of $\mathrm{BM}$ of 4.3 months, followed by Luminal A subtype with 5.2 months, HER2 positive subtype with 9.1 months and Luminal B/HER2 enriched subtype with 11.4 months (Table S2, Figure S2). In addition, TNBC patients had the shortest interval between diagnosis of ECM and the first diagnosis of BM of 5.7 months followed by Luminal A subtype with 14.3 months, HER2 positive subtype 
with 13.8 months and Luminal B/HER2 enriched subtype with 19.3 months. Median survival times in the KPS subgroups differed between 17.9 months (KPS 100\%, 95\% CI 12.9-22.1) and 2.1 months for KPS 10-30\% (1.1-3.0 95\% CI). Patients with the lowest KPS $(10-30 \%)$ had a 4.76 fold increased chance to suffer from an early death (HR 4.76, 95\% CI 2.97-7.63, $p<0.001$ ) compared to those with a KPS of $100 \%$ (Table 3). Multivariate analysis confirmed the association with overall survival for the parameters age $\geq 60$ years, breast cancer subtype, Karnofsky-Performance-Status and number of BM adjusted for ECM (Table S1).

Table 3. Median overall survival after the diagnosis of brain metastases (BM) (univariate analysis).

\begin{tabular}{|c|c|c|c|c|c|c|}
\hline Parameter & Category & $\begin{array}{l}\text { Median } \\
\text { Survival } \\
\text { (months) }\end{array}$ & $95 \%-C I$ & HR & $95 \%$ CI & $p$-Value \\
\hline \multirow[t]{2}{*}{$\begin{array}{c}\text { Age at } \\
\text { diagnosis of } \\
\text { BM, binary }\end{array}$} & $<60$ & 11.3 & $(9.6,13.7)$ & & & \\
\hline & $\geq 60$ & 5 & $(3.9,5.9)$ & 1.53 & $(1.32,1.77)$ & $<0.001$ \\
\hline \multirow[t]{2}{*}{ ECM } & no & 15.6 & $(10.8,23.8)$ & & & \\
\hline & yes & 7.8 & $(6.4,9.0)$ & 1.67 & $(1.34,2.08)$ & $<0.001$ \\
\hline \multirow[t]{3}{*}{ Number of BM } & 1 & 14.1 & $(10.3,19.2)$ & & & \\
\hline & $2-3$ & 9.7 & $(6.1,12.3)$ & 1.34 & $(1.09,1.65)$ & 0.005 \\
\hline & $\geq 4$ & 6.2 & $(5.1,7.2)$ & 1.80 & $(1.50,2.16)$ & $<0.001$ \\
\hline \multirow{4}{*}{$\begin{array}{c}\text { Biological } \\
\text { subtype (acc. } \\
\text { to Sperduto } \\
\text { 2012) }\end{array}$} & TNBC & 4.8 & $(3.8,6.1)$ & & & \\
\hline & $\begin{array}{c}\text { Luminal A } \\
\text { (HR+, } \\
\text { HER2-) }\end{array}$ & 6.0 & $(5.0,7.9)$ & 0.79 & $(0.65,0.95)$ & 0.014 \\
\hline & $\begin{array}{c}\text { Luminal B } \\
(\mathrm{HR}+, \\
\text { HER2+) }\end{array}$ & 16.0 & $(13.0,21.7)$ & 0.43 & $(0.34,0.53)$ & $<0.001$ \\
\hline & $\begin{array}{c}\text { HER2 } \\
\text { (HR-, } \\
\text { HER2+) }\end{array}$ & 12.3 & $(9.2,17.3)$ & 0.49 & $(0.39,0.62)$ & $<0.001$ \\
\hline \multirow[t]{5}{*}{ KPS } & $100 \%$ & 17.9 & $(12.9,22.1)$ & & & \\
\hline & $80-90 \%$ & 13.0 & $(10.8,15.1)$ & 1.26 & $(0.99,1.60)$ & 0.057 \\
\hline & $60-70 \%$ & 4.4 & $(3.5,5.2)$ & 2.28 & $(1.78,2.92)$ & $<0.001$ \\
\hline & $40-50 \%$ & 2.9 & $(2.2,3.9)$ & 3.66 & $(2.68,5.00)$ & $<0.001$ \\
\hline & $10-30 \%$ & 2.1 & $(1.1,3.0)$ & 4.76 & $(2.97,7.63)$ & $<0.001$ \\
\hline
\end{tabular}

\subsection{Prognostic Indices}

In the original GPA, patients were distributed in four categories depending on their point scores $(0-4 \mathrm{p})$. In total, $32.3 \%$ of patients $(n=285)$ were in group $1(0-1 \mathrm{p})$ and $5.2 \%$ $(n=46)$ in group 4 (3.5-4 p). Median survival times in the four groups were 3.7, 10.1, 22.4 and 38.2 months (Table 4, Figure 1).

The median survival times between the four categories of the Breast-GPA score were shown to be at 2.2, 5.4, 8.6 and 21.7 months (Table 4, Figure 2). Only $9.2 \%$ of patients were included in the worst prognostic group $(n=81)$, whereas $27.3 \%$ of patients were included in the best prognostic group $(n=241)$.

In the updated Breast-GPA median survival times variated between 2.7, 5.2, 15.2 and 32.2 months for the four categories (Table 4, Figure 3). All in all, $12.9 \%$ of the patients were contributed to the worst prognostic group $(n=114)$ and only $10.1 \%$ in the best prognostic group $(n=89)$. All categories defined by all three scores were associated with survival in univariate analysis $(p<0.001$ for all scores, Table 4$)$. 
Table 4. Median survival according to the three GPA scores after the diagnosis of BM.

\begin{tabular}{cccccccc}
\hline Parameter & Group & Points & $\begin{array}{c}\text { Number } \\
\text { of Patients }\end{array}$ & $\begin{array}{c}\text { Median } \\
\text { Survival } \\
\text { (months) }\end{array}$ & HR & 95\%-CI & $p$-Value \\
\hline Original & 1 & $0-1$ & 285 & 3.7 & & & \\
GPA & 2 & $1.5-2.5$ & 478 & 10.1 & 0.53 & $(0.46,0.63)$ & $<0.001$ \\
& 3 & 3.0 & 73 & 22.4 & 0.34 & $(0.25,0.46)$ & $<0.001$ \\
& 4 & $3.5-4.0$ & 46 & 38.2 & 0.21 & $(0.14,0.32)$ & $<0.001$ \\
Breast- & 1 & $0-1$ & 81 & 2.2 & & & \\
GPA & 2 & $1.5-2.0$ & 236 & 5.4 & 0.47 & $(0.36,0.61)$ & $<0.001$ \\
& 3 & $2.5-3.0$ & 324 & 8.6 & 0.31 & $(0.24,0.41)$ & $<0.001$ \\
& 4 & $3.5-4.0$ & 241 & 21.7 & 0.17 & $(0.13,0.23)$ & $<0.001$ \\
updated & 1 & $0-1$ & 114 & 2.7 & & & \\
Breast- & 2 & $1.5-2.0$ & 327 & 5.2 & 0.63 & $(0.50,0.78)$ & $<0.001$ \\
GPA & 3 & $2.5-3.0$ & 352 & 15.2 & 0.29 & $(0.23,0.37)$ & $<0.001$ \\
& 4 & $3.5-4.0$ & 89 & 32.2 & 0.15 & $(0.11,0.21)$ & $<0.001$ \\
\hline
\end{tabular}

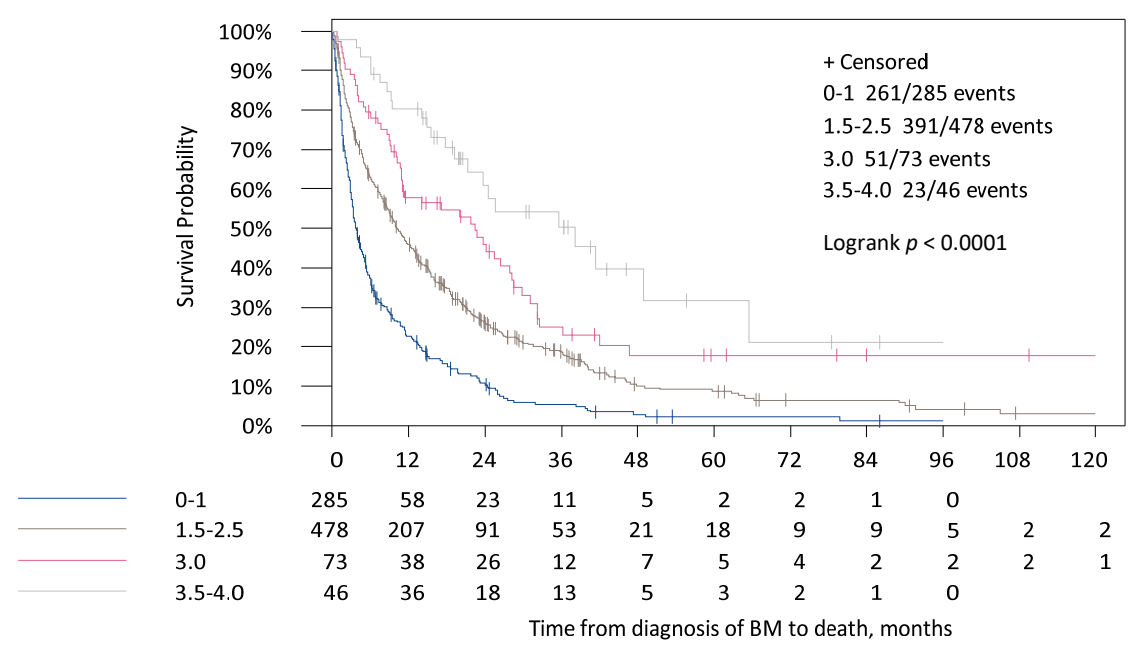

Figure 1. Survival times for the time from diagnosis of BM to death in the four categories of the original GPA Score.

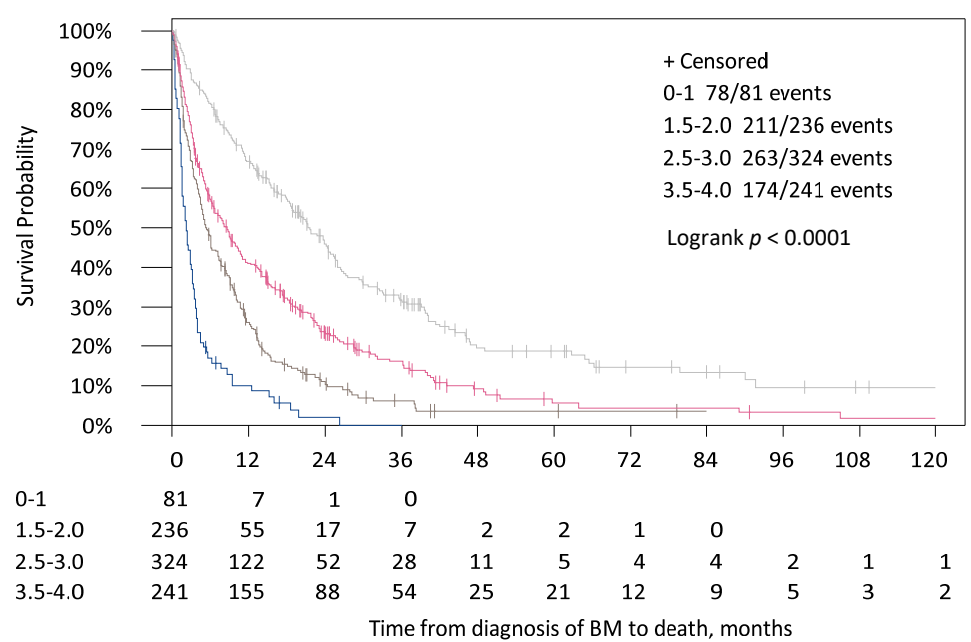

Figure 2. Survival times for the time from diagnosis of BM to death in the four categories of the Breast-GPA Score. 


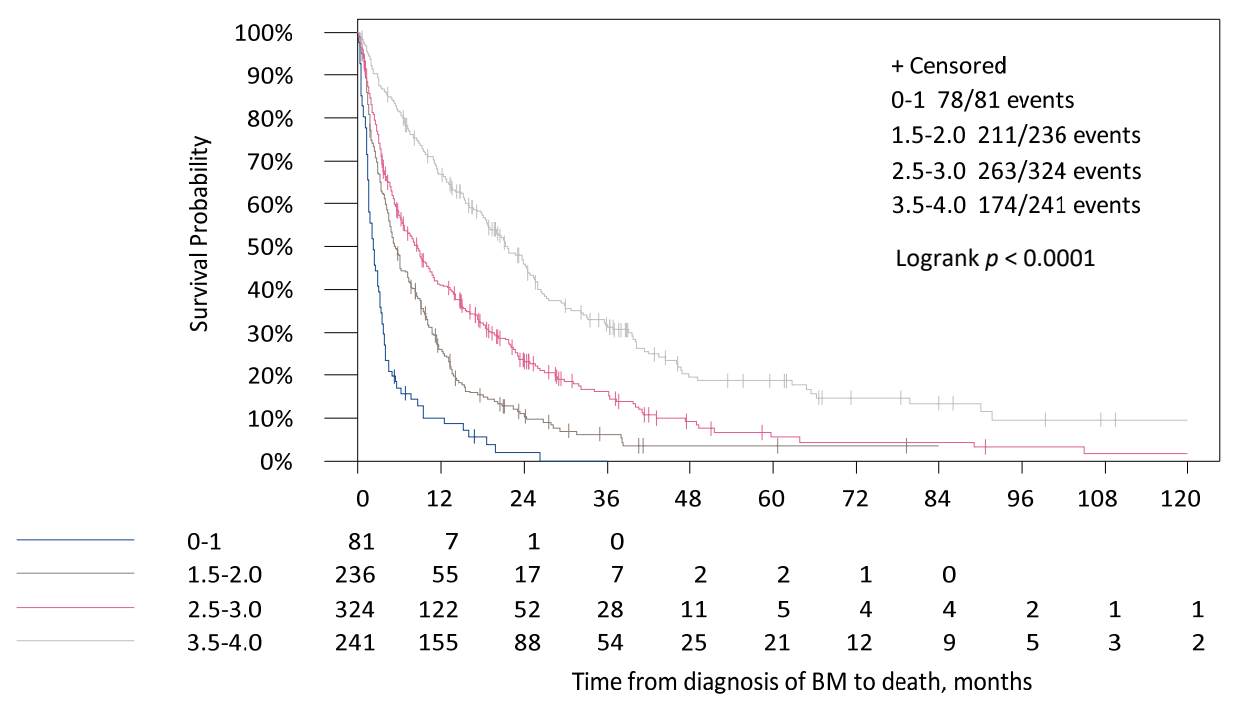

Figure 3. Survival times for the time from diagnosis of BM to death in the four categories of the updated Breast-GPA Score.

\subsection{Diagnostic Accuracy of GPA Scores}

When looking at time-dependent specificities identifying patients with a long-life expectancy ( $>12$ months) the highest category of each score was compared with the lower three categories (Table 5). The Breast-GPA had the highest value of specificity with $68.7 \%$ in comparison to the updated Breast-GPA with $48.1 \%$ and the original GPA with $21.8 \%$. The time-dependent NPV (the probability of living longer than 12 months with a high point score), was slightly higher for the updated Breast-GPA with $69.1 \%$, compared to the original GPA with $66.3 \%$ and the Breast-GPA with $60.2 \%$.

Table 5. Diagnostic accuracy of the original GPA, the Breast-GPA and updated Breast-GPA score at the time of 12 months for a cut off value of 3 , comparing the highest category versus the three lower categories of each score.

\begin{tabular}{cccccc}
\hline GPA-Score & Timepoint & $\begin{array}{c}\text { Time-Dependent } \\
\text { Sensitivity (\%) } \\
\mathbf{( 9 5 \% - C I )}\end{array}$ & $\begin{array}{c}\text { Time-Dependent } \\
\text { Specificity (\%) } \\
\mathbf{( 9 5 \% - C I )}\end{array}$ & $\begin{array}{c}\text { Time-Dependent } \\
\text { PPV (\%) (95\%-CI) }\end{array}$ & $\begin{array}{c}\text { Time-Dependent } \\
\text { NPV (\%) (95\%-CI) }\end{array}$ \\
\hline Original GPA & 12 months & $92.2(89.9,94.6)$ & $21.8(17.4,26.2)$ & $62.6(59.1,66.1)$ & $66.3(57.7,75.0)$ \\
Breast-GPA & 12 months & $68.1(64.0,72.2)$ & $68.7(63.8,73.7)$ & $75.6(71.6,79.6)$ & $60.2(55.4,65.0)$ \\
updated Breast GPA & 12 months & $84.8(81.7,88.0)$ & $48.1(42.8,53.4)$ & $69.9(66.2,73.6)$ & $69.1(63.2,74.9)$ \\
\hline
\end{tabular}

For the identification of patients with a low life expectancy ( $<3$ months) the lowest category was compared with the three higher categories of each score (Table 5). Here, the time-dependent sensitivities for all scores were very low with $24.4 \%$ for the original GPA, $11.5 \%$ for the updated Breast-GPA and $6.8 \%$ for the Breast-GPA. Regarding time-dependent PPV (the probability of living shorter than 3 months with a low point score) the breast specific scores had higher values than the original GPA (62.7\% for the updated Breast-GPA and $61.4 \%$ for the Breast-GPA vs. $51.3 \%$ for the original GPA).

Comparing the Receiver Operating Characteristic (ROC) of the three different scores, the updated Breast-GPA score showed the best results for AUC values of 71.4 for 3 months (95\% CI 67.8-75.0) and 74.2 for 12 months (95\%-CI 70.9-77.5) in comparison to the BreastGPA with AUC values of 69.1 for 3 months (95\%-CI 65.3-73.0) and 73.0 for 12 months (95\%-CI 69.6-76.4) and the original GPA score with AUC values of 70.0 for 3 months (95\%-CI 66.3-73.7) and 69.5 for 12 months (95\%-CI 66.0-73.1).

Nonetheless, there were no significant differences between the AUC of all three scores after 12 months (Table 6). On the contrary, the AUC after 3 months was better for the updated Breast-GPA compared with the Breast-GPA ( $p=0.010$, Table 7). In conclusion, there is a lack of discrimination between all scores by gaining AUC results of around $70 \%$. 
Table 6. Diagnostic accuracy of the original GPA, the Breast-GPA and updated Breast-GPA score at the time of 3 months for a cut off value of 1 , comparing the lowest category versus the three higher categories of each score.

\begin{tabular}{cccccc}
\hline GPA-Score & Time Point & $\begin{array}{c}\text { Time-Dependent } \\
\text { Sensitivity (\%) } \\
\mathbf{( 9 5 \% - C I )}\end{array}$ & $\begin{array}{c}\text { Time-Dependent } \\
\text { Specificity (\%) } \\
\mathbf{( 9 5 \% - C I )}\end{array}$ & $\begin{array}{c}\text { Time-Dependent } \\
\text { PPV (\%) }\end{array}$ & $\begin{array}{c}\text { Time-Dep-CI) } \\
\text { NPV (\%) (95\%-CI) }\end{array}$ \\
\hline Original GPA & 3 months & $24.4(18.9,29.9)$ & $91.6(89.4,93.7)$ & $51.3(42.0,60.6)$ & $76.9(73.9,79.9)$ \\
Breast-GPA & 3 months & $6.8(3.6,10.1)$ & $98.4(97.5,99.4)$ & $61.4(42.7,80.2)$ & $74.4(71.4,77.3)$ \\
updatedBreast-GPA & 3 months & $11.5(7.4,15.6)$ & $97.5(96.3,98.7)$ & $62.7(48.3,77.2)$ & $75.2(72.2,78.1)$ \\
\hline
\end{tabular}

Table 7. Area under the time-dependent Receiver Operating Characteristic (ROC) curve (AUC) with the corresponding 95\%-CI for all three GPA-Scores for two different time points ( 3 and 12 months).

\begin{tabular}{|c|c|c|c|c|}
\hline GPA-Score & Time Point & $\begin{array}{l}\text { AUC (\%) } \\
(95 \%-C I)\end{array}$ & Comparison & $p$-Value \\
\hline Original GPA & 3 months & $70.0(66.3,73.7)$ & \multirow{4}{*}{ Breast-GPA vs. original GPA } & \\
\hline Original GPA & 12 months & $69.5(66.0,73.1)$ & & \\
\hline Breast-GPA & 3 months & $69.1(65.3,73.0)$ & & 0.698 \\
\hline Breast-GPA & 12 months & $73.0(69.6,76.4)$ & & 0.086 \\
\hline updated Breast-GPA & 3 months & $71.4(67.8,75.0)$ & \multirow{2}{*}{$\begin{array}{c}\text { Breast-GPA vs. updated } \\
\text { Breast-GPA }\end{array}$} & 0.010 \\
\hline updated Breast-GPA & 12 months & $74.2(70.9,77.5)$ & & 0.176 \\
\hline
\end{tabular}

\section{Discussion}

Treatment of BM usually involves local and systemic treatment [2]. In almost all cases, local therapy consists of radiotherapy with or without neurosurgery. Despite improved local intracranial control, radiotherapy of the brain did not show improved survival in BC patients and, instead, leads to certain toxicities [11]. Recently, new agents might have also improved survival times in a subset of HER2 positive BC patients with BM [12] and some trials also focus on patients with BM of HER2 negative breast cancer [13]. In order to help to identify patients with good and bad prognosis easily, different scores were developed combining different prognostic parameters [4]. All tests were developed with patients with $\mathrm{BM}$ who had received radiotherapy of the brain. In comparison, $14 \%$ of patients in our cohort did not receive radiotherapy or local treatment of the brain which might explain the shorter survival times in our cohort in comparison to original cohorts [8]. However, the median OS time of 8.7 months is in the range of published real-world data of $\mathrm{BC}$ patients with BM [14].

It remains difficult to predict the prognosis of $\mathrm{BC}$ patients with $\mathrm{BM}$, although several risk factors have already been identified that are associated with impaired survival. Scores should help to stratify local and systemic treatment according to the patient's prognosis. As age, KPS and ECM are associated with survival in patients with BM, these parameters were used to calculate the GPA Score in 2008 [6]. To further improve the test accuracy, the breast-specific scores Breast-GPA and updated Breast-GPA were introduced which include tumour subtype [8,9]. In both breast-specific scores, the luminal-B/HER2 enriched group was established as a separate category. In our analysis, patients with HER2 positive, hormone receptor-positive (triple positive) $\mathrm{BC}$ had the best prognosis compared to HER2 positive, hormone receptor-negative and HER2 negative BC. Although triple positivity reveals resistance to HER2-directed treatment in the adjuvant or neoadjuvant setting [15], the prognosis of patients with BM is superior to other subtypes [16,17], thus, supporting the classification as own category in patients with BM. The improved survival of HER2-positive patients is often explained by the possibility of agents suitable for passing the blood-brain barrier [12].

All three GPA scores were associated with OS in our analysis. However, we could show that breast-specific GPA scores which include tumour subtype in the calculation of the score performed slightly better than the original GPA score. However, all scores had a 
rather low test accuracy in our analysis. The breast-GPA with a specificity of $68.7 \%$ and an NPV of $66.3 \%$ for 12-months survival could filter the long-term survivors from the best prognostic group as well as patients with a bad prognosis from the lowest prognostic group (PPV: 61.4\%, sensitivity: 6.8\%). A high PPV is an important quality factor mainly for the lowest prognostic group in order to prevent patients from getting misleadingly selected in the worst prognosis group and therefore being held from potentially effective therapy [18]. Due to the same reason, the number of patients in the lowest prognostic group should be kept as small as possible [19]. Although the original GPA had the highest sensitivity (24.4\%) for predicting 3-months survival, it categorized almost one-third of patients into the lowest category (32.3\%). In contrast, the Breast-GPA and the updated Breast-GPA categorized a smaller number of patients into the lowest category $(9.2 \%$ and $13 \%)$. In line with published data, the breast-specific scores had higher PPV and performed slightly better in predicting short survival below 3 months than the original GPA [4]. In addition, the Breast-GPA assigned the highest percentage of patients in the best prognostic group $(27.3 \%)$ in comparison to the updated Breast-GPA $(10.1 \%)$ and the original GPA $(5.2 \%)$. This could explain the shorter median survival time for the breast-GPA patients of the best prognostic group with 21.7 months (original GPA 38.2 and updated BreastGPA 32.2 months). The breast-GPA also had the highest specificity (68.7\%) in identifying patients with a long life expectancy ( $>12$ months). It needs to be discussed whether the best prognostic group should separate only a minority of patients with an excellent prognosis of more than 3 years or a larger group with also a very good prognosis of around 2 years for selecting therapy options.

\section{Materials and Methods}

The BMBC registry is a multicentre trial evaluating clinical data of BC patients with BM run by the German Breast Group (GBG), the Translation Research Board and the Breast Study Group of the Working Group Gynaecologic Oncology Germany (AGO-Trafo and AGO-B) and the University Medical Center Hamburg, Germany. Patients were identified retrospectively as well as prospectively if they had a diagnosis of BM based on appropriate imaging and/or histological findings since the year 2000 and a history of BC. Patients were excluded if they had a history of other malignant diseases, no histological verification of the diagnosis of $\mathrm{BC}$, a history of neurologic disease or leptomeningeal disease without solid BM. By August 2019, 105 study sites had documented clinical data of 2589 patients. In sum, 1158 patients (44.73\%) were treated in a university setting, 1431 (55.27\%) in a non-university setting. All participating study sites were either located in departments of gynaecology or medical oncology. The BMBC registry was approved by all local ethics committees. For 882 (34\%) from overall 2589 patients, all three GPA scores could be determined and therefore were considered eligible for this study.

\subsection{Calculation of GPA Scores}

In the original GPA score, four groups reflecting median survival times were calculated on the basis of the prognostic factors KPS, number of BM, ECM and age at the first diagnosis of BM. In the Breast-GPA number of BM and ECM were replaced by tumour subtype and also four median survival groups were estimated. In the updated version of the Breast-GPA score presence of ECM was added to the parameters already included in the Breast-GPA Score and four survival groups were calculated. For parameters and calculation of the GPA Scores, see Table 1.

\subsection{Biological Subtype and ECOG/Karnofsky Performance Status}

In order to calculate the disease-specific GPA scores tumour subtypes had to be redefined as HER2 positive including only estrogen receptor (ER) and/or progesterone receptor (PR) negative and HER2 positive tumours and Luminal B-HER2 enriched including HER2 positive and ER and/or PR positive tumours. Luminal A was defined as ER-positive, HER2 negative and triple-negative as ER-negative and HER2 negative. 
ECOG/Karnofsky performance status was categorized in $0(100 \%), 1(80-90 \%), 2$ (60-70\%), $3(40-50 \%), 4(10-30 \%)$ and unknown.

\subsection{Statistical Analysis}

Continuous data were summarized using the number of available data, mean, standard deviation (SD), median, minimum and maximum for each group. Categorical and ordinal data were summarized using the number and percentage of patients in each group.

Further, Kaplan-Meier curves and the median OS time with the corresponding 95\% confidence interval $(\mathrm{CI})$ were determined to assess the association of the OS with the survival times of several factors (age at first diagnosis of BM, number of BM, biological subtype, KPS, appearance of ECM) and of the three prognostic scores. OS was defined as the time interval from the first diagnosis of BM to death due to any reason. Differences in the survival curves were tested by the log-rank test. All reported $p$-values are two-sided, and the significance level was set to 0.05 . Confidence intervals symmetrically cover $95 \%$.

The data were analyzed using SAS®(Statistical Analysis Software) version 9.4 (SAS Institute Inc., Cary, NC, USA)with SAS Enterprise Guide Version 7.1 on Microsoft Windows 10 Enterprise (Microsoft Corporation, Redmond, WA, USA). The diagnostic accuracy of the Breast-GPA, the updated Breast-GPA and the original GPA score was described by timedependent sensitivities, specificities, positive and negative predictive values (PPV; NPV) at the time points after 3 months to identify a short life expectancy and after 12 months to identify a long life expectancy for the selected cut-off values. For the identification of a short life expectancy ( $<3$ months) a high score of sensitivity and PPV was defined as the best factors for excellent test quality, whereas for the identification of a long life expectancy (>12 months), a high specificity and high NPV were defined as best factors for excellent test quality. Cut-offs for short- and long-term survival were used in order to help identify patients both eligible for extensive treatment and spare patients with worse outcomes from overtreatment. Additionally, the corresponding $95 \%$-CI of these time-dependent measures were determined.

Furthermore, as a measure of accuracy, the areas under the time-dependent Receiver Operating Characteristic (ROC) curves (AUC) were determined. In the time-dependent ROC curves, the sensitivities were plotted against the specificities for different cut-off values of the original score, the Breast-GPA and the updated Breast-GPA at the time of 3 and 12 months.

The analyses of the described time-dependent measures were performed using $\mathrm{R}$ from the R Foundation for Statistical Computing, Vienna, Austria (version 3.6.0), particularly the R package time ROC by P. Blanche (version 0.3) [20].

\section{Conclusions}

In summary, we could show in a real-world cohort of BC patients with BM that although all GPA-Scores were associated with overall survival, the addition of diseasespecific parameters resulted in better test accuracy. However, adding those parameters did not improve the accuracy as much as expected. All currently available prognostic scores show limitations mainly in predicting short-term (below 3 months) survival but also in long-term (above 12 months) survival and, thus, should be employed carefully by physicians when being used for further therapy decisions. Further studies should focus on the identification of biomarkers that might help to improve estimating the prognosis of $\mathrm{BC}$ patients with BM.

Supplementary Materials: The following are available online at https:/ / www.mdpi.com/2072-6 694/13/4/844/s1. Table S1: Multivariate Cox regression analysis of the time from BM to death, Table S2: Progression free Survival according to Breast Cancer subtype, Table S3: Distribution of the subtype (according to Sperduto 2012) in patients with meningeosis carcinomatosa ( $N=252$ of overall 2589 patients), Table S4: Local treatment according to subtype (Sperduto 2012), Table S5: Distribution of BC treatment between different subtypes after the diagnosis of BM, Table S6: specification of BC 
treatment after the diagnosis of BM, Figure S1: Overall Survival for Leptomeningeal disease and solid BM vs. only solid BM, Figure S2: Time from first diagnosis of BM to progress

Author Contributions: Conceptualization, K.R., V.M. (Volkmar Müller), E.L. and I.W.; Formal analysis, J.R.; Methodology, K.R., E.L., I.W.; Project administration, I.W.; Software, V.N, J.R.; Supervision, V.M. (Volkmar Müller), E.L., I.W.; Validation, K.R., V.M. (Volkmar Müller), V.N., J.R., I.W.; Visualization, V.N. and J.R.; Writing-original draft, K.R. and I.W.; Writing-review \& editing, K.R., V.M. (Volkmar Müller), R.W., M.S., T.-W.P.-S., V.M. (Volker Möbus), C.M., A.P., K.L., T.H., E.L., M.T., P.A.F., C.D., T.F., V.N., J.R., S.L., I.W. All authors have read and agreed to the published version of the manuscript.

Funding: This research received no external funding.

Institutional Review Board Statement: The clinical data for the analysis was derived from the BMBC Registry. Patients with BM of breast cancer registered until April 2019 were considered for this analysis. The BMBC registry was approved by the ethic committee Hessen, Frankfurt am Main (ethic approval number FF42/2013).

Informed Consent Statement: Informed consent was obtained from subjects involved in the study, as defined in the protocol.

Data Availability Statement: Data is contained within the article or Supplementary Material.

Conflicts of Interest: The authors declare no conflict of interest.

\section{References}

1. Smedby, K.E.; Brandt, L.; Bäcklund, M.L.; Blomqvist, P. Brain metastases admissions in Sweden between 1987 and 2006. Br. J. Cancer 2009, 101, 1919-1924. [CrossRef] [PubMed]

2. Witzel, I.; Oliveira-Ferrer, L.; Pantel, K.; Muller, V.; Wikman, H. Breast cancer brain metastases: Biology and new clinical perspectives. Breast Cancer Res. 2016, 18, 8. [CrossRef] [PubMed]

3. Witzel, I.; Laakmann, E.; Weide, R.; Neunhöffer, T.; Park-Simon, T.-J.; Schmidt, M.; Fasching, P.; Hesse, T.; Polasik, A.; Mohrmann, S.; et al. Treatment and outcomes of patients in the Brain Metastases in Breast Cancer Network Registry. Eur. J. Cancer 2018, 102, 1-9. [CrossRef] [PubMed]

4. Laakmann, E.; Riecke, K.; Goy, Y.; Kersten, J.F.; Krüll, A.; Müller, V.; Petersen, C.; Witzel, I. Comparison of nine prognostic scores in patients with brain metastases of breast cancer receiving radiotherapy of the brain. J. Cancer Res. Clin. Oncol. 2015, 142, 325-332. [CrossRef] [PubMed]

5. Li, J.; Bentzen, S.M.; Li, J.; Renschler, M.F.; Mehta, M.P. Relationship between neurocognitive function and quality of life after whole-brain radiotherapy in patients with brain metastasis. Int. J. Radiat. Oncol. 2008, 71, 64-70. [CrossRef] [PubMed]

6. Sperduto, P.W.; Berkey, B.; Gaspar, L.E.; Mehta, M.; Curran, W. A new prognostic index and comparison to three other indices for patients with brain metastases: An analysis of 1960 patients in the RTOG database. Int. J. Radiat. Oncol. 2008, 70, 510-514. [CrossRef] [PubMed]

7. Antoni, D.; Clavier, J.-B.; Pop, M.; Schumacher, C.; Lefebvre, F.; Noel, G. Institutional, retrospective analysis of 777 patients with brain metastases: Treatment outcomes and diagnosis-specific prognostic factors. Int. J. Radiat. Oncol. 2013, 86, 630-637. [CrossRef] [PubMed]

8. Sperduto, P.W.; Kased, N.; Roberge, D.; Xu, Z.; Shanley, R.; Luo, X.; Sneed, P.K.; Chao, S.T.; Weil, R.J.; Suh, J.; et al. Effect of tumor subtype on survival and the graded prognostic assessment for patients with breast cancer and brain metastases. Int. J. Radiat. Oncol. 2012, 82, 2111-2117. [CrossRef] [PubMed]

9. Sperduto, P.W.; Mesko, S.; Li, J.; Cagney, D.; Aizer, A.; Lin, N.U.; Nesbit, E.; Kruser, T.J.; Chan, J.; Braunstein, S.; et al. Beyond an updated graded prognostic assessment (Breast GPA): A prognostic index and trends in treatment and survival in breast cancer brain metastases from 1985 to today. Int. J. Radiat. Oncol. 2020, 107, 334-343. [CrossRef] [PubMed]

10. Tabouret, E.; Metellus, P.; Gonçalves, A.; Esterni, B.; Charaffe-Jauffret, E.; Viens, P.; Tallet, A. Assessment of prognostic scores in brain metastases from breast cancer. Neuro Oncol. 2014, 16, 421-428. [CrossRef] [PubMed]

11. Brown, P.D.; Ahluwalia, M.S.; Khan, O.H.; Asher, A.L.; Wefel, J.S.; Gondi, V. Whole-brain radiotherapy for brain metastases: Evolution or revolution? J. Clin. Oncol. 2018, 36, 483-491. [CrossRef] [PubMed]

12. Lin, N.U.; Borges, V.; Anders, C.; Murthy, R.K.; Paplomata, E.; Hamilton, E.; Hurvitz, S.; Loi, S.; Okines, A.; Abramson, V.; et al. Intracranial efficacy and survival with tucatinib plus trastuzumab and capecitabine for previously treated HER2-positive breast cancer with brain metastases in the HER2CLIMB trial. J. Clin. Oncol. 2020, 38, 2610-2619. [CrossRef] [PubMed]

13. Perez, E.A.; Awada, A.; O'Shaughnessy, J.; Rugo, H.S.; Twelves, C.; Im, S.A.; Gomez-Pardo, P.; Schwartzberg, L.S.; Dieras, V.; Yardley, D.A.; et al. Etirinotecan pegol (NKTR-102) versus treatment of physician's choice in women with advanced breast cancer previously treated with an anthracycline, a taxane, and capecitabine (BEACON): A randomised, open-label, multicentre, phase 3 trial. Lancet Oncol. 2015, 16, 1556-1568. [CrossRef] 
14. Darlix, A.; Louvel, G.; Fraisse, J.; Jacot, W.; Brain, E.; Debled, M.; Mouret-Reynier, M.A.; Goncalves, A.; Dalenc, F.; Delaloge, S.; et al. Impact of breast cancer molecular subtypes on the incidence, kinetics and prognosis of central nervous system metastases in a large multicentre real-life cohort. Br. J. Cancer 2019, 121, 991-1000. [CrossRef] [PubMed]

15. Gianni, L.; Pienkowski, T.; Im, Y.-H.; Roman, L.; Tseng, L.-M.; Liu, M.-C.; Lluch, A.; Staroslawska, E.; De La Haba-Rodriguez, J.; Im, S.-A.; et al. Efficacy and safety of neoadjuvant pertuzumab and trastuzumab in women with locally advanced, inflammatory, or early HER2-positive breast cancer (NeoSphere): A randomised multicentre, open-label, phase 2 trial. Lancet Oncol. 2012, 13, 25-32. [CrossRef]

16. Sperduto, P.W.; Kased, N.; Roberge, D.; Chao, S.T.; Shanley, R.; Luo, X.; Sneed, P.K.; Suh, J.; Weil, R.J.; Jensen, A.W.; et al. The effect of tumor subtype on the time from primary diagnosis to development of brain metastases and survival in patients with breast cancer. J. Neurooncol. 2013, 112, 467-472. [CrossRef] [PubMed]

17. Melisko, M.E.; Moore, D.H.; Sneed, P.K.; De Franco, J.; Rugo, H.S. Brain metastases in breast cancer: Clinical and pathologic characteristics associated with improvements in survival. J. Neurooncol. 2008, 88, 359-365. [CrossRef] [PubMed]

18. Zindler, J.D.; Rodrigues, G.; Haasbeek, C.J.; De Haan, P.F.; Meijer, O.W.; Slotman, B.J.; Lagerwaard, F.J. The clinical utility of prognostic scoring systems in patients with brain metastases treated with radiosurgery. Radiother. Oncol. 2013, 106, 370-374. [CrossRef] [PubMed]

19. Nieder, C.; Marienhagen, K.; Thamm, R.; Astner, S.; Molls, M.; Norum, J. Prediction of very short survival in patients with brain metastases from breast cancer. Clin. Oncol. 2008, 20, 337-339. [CrossRef] [PubMed]

20. Blanche, P.; Dartigues, J.F.; Jacqmin-Gadda, H. Estimating and comparing time-dependent areas under receiver operating characteristic curves for censored event times with competing risks. Stat. Med. 2013, 32, 5381-5397. [CrossRef] [PubMed] 